\title{
Les facteurs d'influence de la participation verbale en classe des étudiants universitaires : une revue de la littérature
}

Anastassis Kozanitis et Roch Chouinard

\section{(2) OpenEdition \\ Journals}

Édition électronique

URL : http://journals.openedition.org/ripes/59

DOI : $10.4000 /$ ripes.59

ISSN : 2076-8427

Éditeur

Association internationale de pédagogie universitaire

Référence électronique

Anastassis Kozanitis et Roch Chouinard, « Les facteurs d'influence de la participation verbale en classe des étudiants universitaires : une revue de la littérature ", Revue internationale de pédagogie de l'enseignement supérieur [En ligne], 25(1) | 2009, mis en ligne le 20 avril 2009, consulté le 10 décembre 2020. URL : http://journals.openedition.org/ripes/59 ; DOI : https://doi.org/10.4000/ripes.59

Ce document a été généré automatiquement le 10 décembre 2020.

Article L.111-1 du Code de la propriété intellectuelle. 


\title{
Les facteurs d'influence de la participation verbale en classe des étudiants universitaires : une revue de la littérature
}

\author{
Anastassis Kozanitis et Roch Chouinard
}

\section{Introduction}

1 Depuis les années 1980, plusieurs chercheurs se sont intéressés aux facteurs de réussite et d'échec à l'université, et plus largement aux facteurs et indicateurs d'une formation de qualité. (Astin, 1984, 1993 ; Chickerin et Gamson, 1987 ; Pascarella et Terenzini, 1991 ; Smart, Feldman et Ethington, 2000). Ces auteurs affirment que la qualité d'un apprentissage n'est pas seulement liée à des facteurs d'ordre cognitif mais aussi à des facteurs d'ordre affectif. À ce propos, on s'est particulièrement intéressé à la relation pédagogique et à la qualité de l'engagement des étudiants dans leurs études. Ramsden (2003) souligne que l'aspect émotionnel de la relation pédagogique compte davantage que tout autre élément relatif aux méthodes ou aux techniques d'enseignement. De plus, cet auteur affirme que les étudiants ont de meilleures chances de comprendre le contenu d'un exposé lorsque l'enseignant interagit avec eux. Il ajoute que cela encourage leur engagement dans le cours et suscite leur intérêt pour la matière. Dans le milieu universitaire, l'engagement dans les études est considéré comme un facteur prépondérant en matière de persévérance et de réussite (Bradley et Graham, 2000 ; Conseil supérieur de l'éducation, 2000 ; House, 2000 et Kuh, 2001).

2 Ainsi, certains auteurs suggèrent que les interactions de nature verbale entre enseignant $^{1}$ et étudiants représentent l'aspect le plus prégnant de l'engagement en ce qui a trait à la qualité de l'apprentissage et de la persévérance (Astin, 1984 ; Terenzini, Pascarella et Blimling, 1996 ; Willis 1993). En effet, entretenir des interactions fréquentes avec les enseignants semble être l'élément qui contribue le plus fortement à 
la satisfaction envers l'expérience universitaire (Crombie, Pyke, Silverthorn, Jones et Piccinin, 2003 ; DeLucia, 1994 ; Lamport, 1993 ; Pascarella et Terenzini, 1991). Cela contribue également à l'apprentissage et à la réussite des études (Astin, 1984, 1993; Chickering et Ehrmann, 1996; Chickerin et Gamson, 1987; Kuh, Schuh et Whitt, 1991; Pace, 1995).

Cela dit, les interactions peuvent avoir lieu en classe ou à l'extérieur de la classe et impliquer les étudiants, les enseignants ou d'autres membres du personnel. Dans le cadre de la présente revue de littérature, l'attention sera principalement portée sur la notion des interactions en classe, et plus particulièrement sur les interactions verbales qui se produisent entre les étudiants et l'enseignant en situation d'apprentissage. Ce choix s'appuie sur un certain nombre de recherches qui indiquent que des contacts fréquents et significatifs avec les enseignants, spécialement les contacts comportant des échanges orientés vers des aspects intellectuels ou liés à la matière, semblent augmenter la motivation des étudiants et leur engagement par rapport aux études (Astin, 1984 ; Kozanitis, Desbiens et Chouinard, 2007 ; Kubanek et Waller, 1994 ; Parmentier, Gathy, Paquet, De Ketele et Denef, 1991 ; Pirot et De Ketele, 2000 ; Terenzini, Pascarella et Lorang, 1982 ; Tinto, 1987).

4 Pour définir de façon opérationnelle la participation verbale, nous avons retenu le modèle INTERSECT de Sadker, Bauchner, Sadker et Hergert (1981), puis adapté par Canada et Pringle (1995), qui catégorise les échanges selon qu'il s'agit de questions initiées par les étudiants, des réponses des étudiants aux questions de l'enseignant et de commentaires ou d'opinions qu'émettent les étudiants. Nous retenons ces trois instances pour définir cette variable, puis nous en rajoutons une quatrième, soit les discussions qui se déroulent en classe. Un tel choix s'appuie sur des auteurs tels que Reynolds et Nunn (1997), Fassinger (1995a et 1995b), Auster et MacRone (1994), Howard et Henney (1998), Brady et Eilser (1999) et Nunn (1996) qui révèlent que les discussions en classe offrent la chance aux étudiants d'atteindre les niveaux d'habileté élevés dans la taxonomie de Bloom. De plus, cette façon de définir la participation verbale permet de l'étudier dans différents contextes pédagogiques, c'est-à-dire dans des classes où l'enseignant privilégie l'enseignement magistral ou la discussion. Voici les principaux indicateurs qui caractérisent la participation verbale des étudiants :

5 1. L'étudiant pose une question à l'enseignant.

2. L'étudiant répond à une question émise par l'enseignant.

3. L'étudiant fait part de son opinion à propos d'un sujet.

4. L'étudiant prend part à une discussion en classe.

6 De manière générale, nous constatons que les conclusions d'études menées à large échelle se rejoignent. Les études suggèrent que la participation verbale en classe contribue favorablement au processus d'apprentissage. Toutefois, la littérature qui porte sur la participation verbale en contexte universitaire montre avec insistance que celle-ci n'occupe qu'une très faible partie du temps de classe, particulièrement en ce qui a trait au premier cycle (Auster et MacRone, 1994 ; Aitken et Neer, 1992 et 1993 ; Fassinger, 1995a, 1995b ; Howard, Short et Clark, 1996 ; Nunn, 1996). En effet, la participation verbale des étudiants occupe une très petite portion du temps de classe (Barnes, 1983 et Nunn, 1996). Fassinger (1995a) ajoute que seul un petit nombre d'étudiants occupe la majorité du temps des échanges dans une classe. Conséquemment, les étudiants interviennent rarement durant un cours et ils posent peu de questions. Dillon (1988, p. 24), dans son résumé d'une analyse étendue 
d'ouvrages sur les questions posées par les élèves des niveaux primaire et secondaire, écrit que «personne n'a jamais effectué d'étude dans les classes qui montre que les étudiants posent beaucoup de questions». Lors d'observations effectuées dans 27 classes auprès de 721 élèves en dernière année du secondaire, Dillon (1988) a entendu moins d'un pour cent d'entre eux poser des questions dans le but d'obtenir des explications. Les élèves abordent donc leurs études collégiales avec peu d'expérience pour ce qui est de poser des questions (Kubanek et Waller, 1994). Qui plus est, des études récentes, menées en milieu universitaire, abondent dans le même sens et laissent entendre que les étudiants de premier cycle posent très peu de questions en classe (Levin et Karl-Heinz, 2003 ainsi que Paoletti et Rigutti, 2003). De plus, ces auteurs ont constaté que les questions posées correspondent en majorité à des questions de notions factuelles plutôt que des questions d'application ou de compréhension.

7 Somme toute, les échanges verbaux n'occupent qu'une faible partie du temps de la classe et on retrouve, semble-t-il, récurrence des mêmes étudiants qui interviennent. Par ailleurs, la plupart des enseignants ayant pris part aux études de Fassinger (1995b), Morgenstern (1992), Nunn (1996), Kozanitis (1997) et Fritschner (2000), ont dévoilé qu'ils souhaitent un engagement réel des étudiants dans leur propre formation et que se produisent davantage d'échanges verbaux en classe. Ces enseignants désirent que les étudiants s'intéressent à la matière et qu'ils démontrent leur intérêt en posant des questions et en émettant des commentaires. Ce point de vue est également partagé par plusieurs étudiants qui considèrent que la participation verbale est un élément essentiel au processus d'apprentissage et qu'elle devrait occuper une plus grande place durant les cours (Bujold et Saint-Pierre, 1996 ; Fritschner, 2000 ainsi que Reynolds et Nunn, 1997). Ce constat a amené plusieurs chercheurs en pédagogie universitaire à s'intéresser aux facteurs d'influence de la participation verbale en classe des étudiants de premier cycle universitaire. Cet article propose d'explorer la littérature pertinente sur le sujet et tente de répondre à la question suivante: quels sont les facteurs qui influencent la participation verbale des étudiants en classe?

8 Avant d'examiner en détail les ouvrages retenus, il semble pertinent d'indiquer brièvement le processus de recherche et de sélection de la revue bibliographique. De la sorte, la recherche documentaire a été menée par ordinateur à l'intérieur des trois bases de données les plus couramment utilisées par les chercheurs en éducation (ERIC, PsychINFO et Dissertation Abstracts Online). Les paramètres de recherche incluent les manuscrits en langue française et anglaise, publiés depuis l'année 1966. La recherche a d'abord été menée en utilisant les mots clés suivants : «verbal participation », « classroom participation » et «student-teacher interactions ». Toutefois, compte tenu d'une abondante littérature sur le thème, il a été nécessaire de raffiner davantage les mots-clés employés en limitant la recherche aux études menées en milieu universitaire ou collégial. Pour ce, nous avons effectué une deuxième série de recherche en croisant les cibles pertinentes retenues avec les termes " university ", « college » et " postsecondary ». Cela a permis de réduire le corpus de recherche à une soixantaine de documents.

9 Les sections qui suivent permettent de mettre en lumière les résultats les plus éloquents des études qui portent sur les facteurs d'influence de la participation verbale en enseignement supérieur. Soulignons, dans un premier temps, quelques points saillants qui émanent d'un survol de l'ensemble des recherches et qui aideront, à la fois, à faire la synthèse des résultats et de mettre en perspective la portée de ces derniers. 
10 Ainsi, on constate que la majorité des résultats obtenus proviennent d'études menées principalement en milieu anglo-saxon, notamment dans de petits collèges aux ÉtatsUnis. Quelques recherches ont été réalisées au sein de grandes universités nordaméricaines ou européennes. Par contre, les études en milieu universitaire québécois sont quasi inexistantes. Étant donné un contexte culturel différent de celui du milieu anglo-saxon, il serait hasardeux de généraliser les résultats provenant de ces études à la population étudiante québécoise. Puis, on discerne une origine variée des écrits recensés en termes de méthodologies utilisées et de paradigmes sur lesquelles ces recherches reposent. Néanmoins, comme on le verra, les travaux proviennent principalement de trois domaines de recherche, soit en communication, en éducation et en psychologie. Enfin, il faut noter que les études montrent que le niveau de participation verbale des étudiants varie selon plusieurs facteurs. Toutefois, ces études n'offrent que des éléments de réponses à notre question, puisque chaque étude porte sur un ou deux facteurs d'influence de la participation verbale. En effet, peu d'auteurs mettent en relation un plus grand nombre de facteurs. De la sorte, nous avons réparti ces études en trois catégories : celles qui accordent la priorité à l'étudiant (âge, sexe), celles qui se centrent sur l'enseignant (méthode d'enseignement, comportements en classe) et celles qui mettent l'accent sur l'environnement d'apprentissage (taille du groupe classe, aménagement physique de la classe). Cette catégorisation est une proposition originale des auteurs et sert uniquement à faciliter la présentation des résultats. Elle ne suppose pas a priori un quelconque ordre d'importance ou de relations particulières entre les facteurs. En effet, cette revue de littérature ne permet pas d'évaluer l'importance relative des facteurs. Il faudrait, pour cela, réaliser une étude qui tienne compte de l'ensemble des variables afin de faire ressortir les liens entre elles. C'est donc à titre accessoire que nous proposons une organisation en trois catégories des facteurs d'influence, en présentant d'abord les caractéristiques de l'étudiant, puis les variables propres à l'enseignant et en terminant avec les variables en lien avec l'environnement d'apprentissage.

\section{Les caractéristiques de l'étudiant}

11 Les études qui se sont intéressées aux variables propres aux étudiants révèlent que le sexe et l'âge peuvent avoir une influence sur les taux de participation verbale. Par ailleurs, des variables motivationnelles comme l'intérêt pour la matière du cours et la confiance en soi peuvent également jouer un rôle significatif sur la participation verbale. On note que l'influence du sexe des étudiants a fait l'objet des premières études à s'être penchées sur la participation verbale au cours des trente dernières années. Cependant, il émerge de ces études des résultats contradictoires quant au rôle de l'appartenance sexuelle des étudiants. Afin de mieux cerner l'effet du sexe des étudiants sur la participation verbale, il est approprié de considérer ces résultats en les situant dans une perspective historique, en faisant un lien avec les objectifs d'accessibilité de l'enseignement supérieur fixés par le rapport Parent. En effet, la démocratisation de l'enseignement a eu comme effet de changer considérablement la population étudiante des universités, elle n'est plus tout à fait la même qu'on pouvait observer il y a vingt-cinq ou trente ans (Romainville, 2000). Les objectifs d'accessibilité ont introduit dans le système une plus grande hétérogénéité des effectifs étudiants, particulièrement en ce qui a trait au sexe des étudiants. Ainsi, l'accession des femmes à l'enseignement supérieur constitue sans aucun doute un facteur prépondérant de la 
diversification de la population étudiante. À l'université, non seulement a-t-on assisté à une croissance quantitative de la présence des femmes, mais encore observe-t-on une évolution dans la répartition des inscriptions dans les différents programmes d'études. À l'automne 2001, il y a globalement 60 femmes pour 40 hommes dans les universités du Québec. Alors qu'historiquement les hommes étaient majoritaires à l'université, la proportion de femmes n'a cessé d'augmenter au cours des 40 dernières années (Conseil

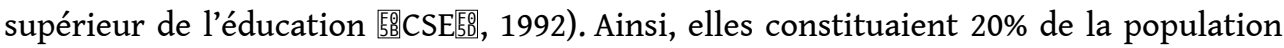
étudiante en $1960,40 \%$ en $1972,50 \%$ en $1980,57 \%$ en 1989 et plus de $60 \%$ en 2001 (Ministère de l'Éducation du Québec 酶MEQ50, 2003). Au premier cycle, les femmes sont majoritaires dans les études à temps partiel et à temps complet. Par ailleurs, il y a maintenant égalité entre hommes et femmes quant à leur nombre dans les études de deuxième cycle. Les hommes seraient, cependant, encore majoritaires dans les programmes de troisième cycle (MEQ, 1995). La répartition des hommes et des femmes dans les divers programmes d'études constitue un autre fait significatif. Ainsi, les femmes sont plus nombreuses dans neuf des dix grands secteurs standardisés: éducation $81 \%$, santé $78 \%$, lettres $74 \%$, arts $65 \%$, sciences humaines $65 \%$, plurisectoriel $65 \%$, droit $61 \%$, sciences pures $56 \%$ et administration $55 \%$. Les hommes ne sont majoritaires que dans le secteur des sciences appliquées où l'évolution du taux d'inscription des femmes (29\%) est plus lente (MEQ, 1995).

À la lumière des recherches consultées, l'évolution du profil de la population étudiante selon le sexe semble correspondre à l'évolution observée quant à l'effet du sexe des étudiants sur la participation verbale. En effet, les études datant des années 70 et 80 révèlent que les hommes prennent plus souvent part aux discussions de classe et posent plus fréquemment des questions que les femmes (Sadker et Sadker, 1986 ; Sternglanz et Lyberger-Ficek, 1977). Le rapport de Hall et Sandler (1982) a mis en évidence le déséquilibre au niveau de l'attention des enseignants portée envers les étudiants, celui-ci étant en faveur des hommes et se caractérise par des échanges verbaux plus fréquents avec ces derniers. Ces auteures ont donné l'appellation en anglais de «chilly classroom climate» pour décrire les classes qui avantagent les étudiants au détriment des étudiantes. Ce rapport a eu un impact important puisque suite à sa parution plusieurs chercheurs se sont intéressés à la question de la participation verbale selon le sexe des étudiants (Canada et Pringle, 1995 ; Cornelius, Gray et Constantinople, 1990 ; Howard et Henney, 1998 ; Sadker et Sadker, 1986, 1992). Ces chercheurs proviennent en majorité du domaine de la communication. Leurs résultats dévoilent que les étudiants profitent davantage des périodes de discussion et de questions pour débattre de leurs idées en public. Ceux-ci tentent de contrôler les discussions de classe en imposant leurs idées aux autres. Ce type d'échange serait préjudiciable aux étudiantes puisque celles-ci cherchent la conciliation plutôt que la confrontation. Les étudiantes préféreraient donc taire leurs idées plutôt que de créer un climat de confrontation en classe. D'autre part, Sternglanz et Lyberger-Ficek (1977) ont trouvé que les étudiants répondent aux questions des enseignants et initient des interactions en classe plus souvent que ne le font les étudiantes. De plus, Sadker et Sadker (1986) révèlent que les étudiants reçoivent davantage d'attention que les étudiantes, et ce, aux trois niveaux d'enseignement, primaire, secondaire et universitaire. Cette dernière constatation a aussi été relevée à l'intérieur d'une revue de la littérature effectuée par Chouinard (1999), qui révèle que des différences dans l'attention des enseignants envers les élèves des deux sexes sont notables dès l'école primaire. 

période où les hommes étaient majoritaires à l'université et doivent être nuancées par les résultats d'études plus récentes. En effet, plusieurs disparités, parfois même des contradictions ont été relevées entre les résultats des chercheurs. Ainsi, Howard et Henney (1998) et Canada et Pringle (1995) ont trouvé des taux de participation plus élevés chez les étudiantes. Par contre, ces auteurs notent que l'avantage que détiennent les femmes pour la participation verbale semble s'estomper lorsque le nombre d'étudiants masculins augmente dans la classe. Malgré cela, Brady et Eisler (1999), Pearson et West (1991), Cornelius, Gray et Constantinople (1990) ainsi que Constantinople, Cornelius et Gray (1988) ne rapportent aucune différence significative dans les taux de participation verbale en lien avec le sexe des étudiants, et ce, en dépit de la proportion du nombre d'étudiants masculins. De leur côté, Boersma, Gay, Jones, Morrison et Remick (1981), ont observé un effet croisé du sexe, c'est-à-dire que les étudiantes ont proportionnellement une plus grande quantité d'interactions verbales lorsque l'enseignant est un homme et vice-versa. étudiantes et par les étudiants quant à leur silence en classe (Fassinger, 1995a ; Christensen, Curley, Marquez et Menzel, 1995). Les raisons des étudiantes se rapportent plus souvent à leur ignorance du sujet, à leur perception d'être incapable de formuler leurs idées adéquatement et à la peur de paraître inintelligentes devant les pairs. Les étudiants, de leur côté, demeurent silencieux parce qu'ils disent ne pas s'être préparés adéquatement pour le cours ou par peur que leurs commentaires n'affectent négativement leurs résultats scolaires. Par ailleurs, Kubanek et Waller (1995) ont identifié des facteurs qui influencent la poursuite des études et la confiance en ses capacités de réussir dans le domaine scientifique des étudiantes de niveau collégial. Ces auteurs ont constaté que le fait de pouvoir poser des questions en classe et de recevoir des réponses satisfaisantes joue un rôle crucial dans la persévérance dans les études, la réussite scolaire et la confiance en soi. Les raisons évoquées par les étudiantes pour expliquer l'importance qu'elles accordent aux questions semblent indiquer que, au-delà des simples réponses et explications factuelles attendues, celles-ci posent des questions aux enseignants pour établir une relation d'apprentissage où elles peuvent se sentir à l'aise. Ces dernières interprètent la manière de répondre de l'enseignant et son empressement à donner des explications comme des indices du type de relation possible. En effet, la langue sert à communiquer les idées, mais elle sert aussi à négocier les relations. Dans sa fonction sociale, la communication devient un outil qui peut être utilisé pour nous évaluer les uns les autres. d'autres facteurs qui peuvent modérer l'influence de l'appartenance sexuelle. En effet, ces auteurs considèrent, par exemple, que le nombre d'années de fréquentation universitaire des étudiants (Crawford et Macleod, 1990), le sexe de l'enseignant (Boersma, Gay, Jones Morrison et Remick, 1981) et la composition sexuelle du groupeclasse (Canada et Pringle, 1995) peuvent affecter l'impact du sexe des étudiants sur la participation verbale. Ces derniers auteurs insistent sur la poursuite des recherches en tenant compte de cette variable en la mettant en relation avec d'autres variables pour en déterminer sa contribution réelle.

Outre le sexe, l'âge des étudiants constitue un autre facteur qui contribue à la diversification de l'effectif estudiantin des dernières années. En effet, les 
établissements d'enseignement supérieur accueillent aujourd'hui une population dont la gamme d'âges est beaucoup plus étendue. Au cours des dernières vingt années, la population universitaire de moins de 25 ans a augmenté de $75 \%$, alors que celle de 25 ans et plus a crû de $150 \%$ (CSE, 1992). Le régime d'études fait ressortir une différence d'âge de 10 ans entre les étudiants inscrits à temps plein et ceux inscrits à temps partiel, la moyenne d'âge du premier groupe étant 23,8 ans et celle du second de 33,3 ans (CSE, 1992). Or, les études montrent que l'âge des étudiants est une variable significative lors de la participation verbale en classe. En effet, plusieurs études ont comparé le taux de participation des étudiants qui se trouvent dans la moyenne d'âge d'étudiants de premier cycle (moins de 25 ans) à celui des étudiants plus âgés ( 25 ans et plus). Les résultats obtenus montrent de manière unanime que les étudiants qui se trouvent dans la catégorie des plus âgés participent plus souvent en classe (BishopClark et Lynch, 1992 ; Bradley et Graham, 2000 ; Fritschner, 2000 ; Howard et Henney, 1998 ; Howard, Short et Clark, 1996 ; Kozanitis et Chouinard, 2007 ; Lynch et BishopClark, 1993). De plus, les étudiants plus âgés interviennent souvent à plus d'une reprise lors d'un même cours. Les plus vieux disent être moins sous l'influence des phénomènes tels que la pression venant des pairs ou la peur de se faire juger négativement par les autres (Fritschner, 2000). Ces auteurs concluent que le sexe des étudiants est une variable significative pour la participation verbale mais que la différence entre étudiantes et étudiants s'amoindrit avec l'âge. Enfin, les étudiants plus âgés démontrent plus de comportements d'engagement envers la matière que les plus jeunes, ils se soucient de bien comprendre la matière et n'hésitent pas à poser des questions lorsqu'ils désirent éclaircir un concept (Bujold et Saint-Pierre, 1996).

17 Toutefois, certains auteurs se questionnent sur la pertinence de considérer des variables sociodémographiques comme le sexe et l'âge, sans les mettre en relation avec d'autres variables lorsqu'on essaie de comprendre des phénomènes sociaux comme la participation verbale en classe (voir Pajares, 1997). En effet, ces travaux issus du domaine de la psychologie et de la psychopédagogie, mettent en garde contre des interprétations trop simplistes de phénomènes complexes et appellent à la prudence lorsque des conclusions sont tirées uniquement sur la base de différences entre le genre ou l'âge des étudiants. En ce sens, ils suggèrent la prise en compte de facteurs personnels et sociorelationnels plus variés comme la motivation scolaire, afin d'en dégager des effets médiateurs ou modérateurs.

De la sorte, certaines études notent que les caractéristiques motivationnelles des étudiants peuvent interagir avec les variables sociodémographiques et influencer de façon directe ou indirecte la participation verbale (Auster et MacRone, 1994 ; Chan et McCroskey, 1987 ; Fassinger, 1995a, 1995b ; Pearson et West, 1991). Il faut cependant mentionner que les études qui portent sur l'impact de la motivation scolaire sur la participation verbale sont rares. Quoi qu'il en soit, les caractéristiques motivationnelles renvoient à des variables de nature affective et comprennent notamment la confiance en soi et l'intérêt pour la matière du cours. Ainsi, selon les études précitées, laconfiance en soides étudiants s'avère la variable qui prédit le plus la participation verbale en classe. De plus, il existe selon Plecha (2002), une relation réciproque entre les interactions étudiants-enseignant et la confiance en soi des étudiants. En effet, cet auteur révèle que les étudiants qui passent du temps à discuter avec les enseignants en dehors des heures de cours rapportent de plus hauts taux de confiance en soi. Par ailleurs, Crawford et MacLeod (1990) notent que les étudiants masculins rapportent avoir davantage confiance en soi que ne le font les étudiantes. Par contre, le 
pourcentage d'étudiantes et d'étudiants qui se sentent suffisamment en confiance pour participer en classe augmente avec le nombre d'années d'études universitaires, et ce, particulièrement pour les femmes (Fassinger, 1995a). Enfin, l'intérêt réfère aux perceptions qu'ont les étudiants de la matière du cours et à leurs croyances quant à l'importance et l'utilité du contenu du cours pour eux. Les auteurs notent que les étudiants qui démontrent plus d'intérêt pour la matière posent plus de questions et participent davantage aux discussions en classe (Christensen, Curley, Marquez et Menzel, 1995 ; Karabenick, 2004 ; Karabenick et Sharma, 1994).

En somme, la diversification de la population étudiante et l'enjeu de l'accès aux études supérieures par un plus grand nombre sont devenus des facteurs fondamentaux dans la conjoncture actuelle. Aujourd'hui, il y a davantage d'étudiants plus âgés inscrits aux études de premier cycle. Le sexe et l'âge des étudiants apparaissent comme deux facteurs qui influencent de façon significative la participation verbale en classe. D'une part, l'âge des étudiants semble être une variable modératrice importante puisque les recherches ont noté que, nonobstant le genre, les étudiants plus âgés démontrent des taux de participation verbale plus élevés que les étudiants plus jeunes. D'autre part, la présence accrue d'étudiantes au sein des programmes d'études semble modifier la distribution des échanges qui se produisent en classe, c'est-à-dire que les taux de participation verbale ont tendance à se répartir plus équitablement quand le nombre d'étudiantes et d'étudiants est équivalent. Par ailleurs, certaines inconsistances dans les résultats entre les différentes recherches, notamment celles qui ont trait au sexe des étudiants, peuvent être reliées à l'évolution des caractéristiques de la population étudiante ou même aux effets indirects de variables motivationnelles. Il faudra à l'avenir en tenir davantage compte dans le traitement qui est fait de la problématique qui nous intéresse. Enfin, il se peut aussi qu'elles soient liées à l'influence de facteurs externes à l'étudiant, tels que les variables propres à l'enseignant. Ces dernières feront l'objet de la prochaine section.

\section{Les variables propres à l'enseignant}

Deux variables propres à l'enseignant ont particulièrement attiré l'attention des chercheurs intéressés par la question de la participation verbale en classe universitaire : les comportements en classe et le sexe de l'enseignant. Les études qui se sont penchées sur les comportements de l'enseignant révèlent que ces derniers peuvent encourager la participation verbale des étudiants. À ce sujet, Auster et MacRone (1994) ont comparé les comportements des enseignants dans les classes où les étudiants ont indiqué participer beaucoup, aux comportements des enseignants dans les classes où les étudiants ont indiqué participer peu, afin d'identifier les comportements des enseignants qui encouragent la participation des étudiants. Ils ont identifié sept comportements pouvant encourager la participation verbale en classe des étudiants. Il s'agit de comportements tels que interroger les étudiants lorsque ceux-ci se portent volontaires, interpeller les étudiants par leur nom, démontrer des signes d'approbation et d'intérêt, encourager les étudiants à élaborer davantage leur réponse, donner suffisamment de temps aux étudiants pour répondre, poser des questions de compréhension et d'analyse et interroger les étudiants lorsque ceux-ci ne se portent pas volontaires. Par exemple, près de 85 pour cent des étudiants ont rapporté que les enseignants les interrogent lorsqu'ils se portent volontaires et les interpellent par leur 
nom dans les classes où il $\mathrm{y}$ a un haut taux de participation, alors que seulement 40 pour cent des enseignants le font dans les classes présentant de faibles taux de participation. Par ailleurs, Nunn (1996) ainsi que Reynolds et Nunn (1997) révèlent que les comportements qui encouragent le plus la participation des étudiants sont l'éloge,l'humour, l'utilisation des idées des étudiants, l'instauration d'une atmosphère de classe propice aux échanges et le fait d'appeler les étudiants par leur nom. Par contre, ils ont trouvé que la critique sévère et la réprimande pour des mauvaises réponses découragent la participation verbale. Qui plus est, certains auteurs affirment que les enseignants qui font preuve d'ouverture et de disponibilité envers les étudiants favorisent la participation verbale (Goldstein et Benassi, 1997 ; Christensen et al. 1995 ; Menzel et Carrell, 1999 ; Thayer-Bacon et Bacon, 1996). Cela suppose qu'ils sont réceptifsà ce que les étudiants ont à dire et respectueux envers eux. Ils mettent l'accent sur les processus d'apprentissage et offrent la chance aux étudiants de s'exprimersur ce qu'ils apprennent de façon à ce qu'ils se sentent engagés dans leurs apprentissages.

21 Par contre, certains de ces résultats de recherche sont contestés par d'autres chercheurs qui affirment que les comportements interpersonnels des enseignants ne semblent pas affecter la participation verbale des étudiants (Fassinger, 1995a et 1995b ; Fritschner, 2000). Ces auteurs ont noté que ce sont plutôt des caractéristiques propres aux étudiants qui interviennent de façon décisive lors de la participation verbale. De surcroît, ils considèrent que l'unique lien médiateur entre les comportements des enseignants et la participation verbale se trouve dans le choix des activités d'apprentissage qui permettent les échanges et les interactions.

En examinant les études qui portent sur l'impact du sexe des enseignants sur la participation verbale, nous constatons que les résultats ne sont pas unanimes. D'abord, quelques recherches indiquent que cette variable exerce peu d'effet sur la participation verbale des étudiants (Boersma, Gay, Jones, Morrison et Remick, 1981 ; Cornelius, Gray et Constantinople, 1990 ; Sternglanz et Lyberger-Ficek, 1977). Cependant, quelques différences relatives à la façon de questionner et d'interagir avec les étudiants sont dignes de mention. De ce fait, Pearson et West (1991) révèlent que les enseignants masculins reçoivent plus souvent des questions que leurs collègues féminins. Karp et Yoels (1976) ont trouvé que les enseignants masculins questionnent les étudiants masculins plus souvent qu'ils ne questionnent les étudiantes. Par contre, des études plus récentes révèlent que les enseignantes entretiennent un plus grand nombre d'interactions verbales en classe avec les étudiants en général que leurs collègues masculins (Brady et Eisler, 1999 ; Canada et Pringle, 1995 ainsi que Crawford et MacLeod, 1990). En effet, il semble que les enseignantes interpellent davantage directement les étudiants, posent des questions, utilisent l'humour et encouragent les étudiants plus fréquemment que ne le font les enseignants masculins. Enfin, l'étude de Brady et Eisler (1999) rapporte que les enseignants ont recours aux exposés magistraux plus souvent que leurs collègues féminins, et qu'ils posent moins de questions.

Dans l'ensemble, les études ayant examiné l'impact des variables en lien avec l'enseignant sur la participation verbale montrent que les comportements des enseignants susceptibles de répondre aux besoins de sécurité des étudiants sont les plus susceptibles d'encourager la participation verbale. De plus, les recherches plus récentes suggèrent que ce sont surtout les enseignantes qui ont recours à ces comportements le plus fréquemment. Cependant, malgré les efforts des enseignants pour encourager la participation verbale en classe, certains facteurs relatifs à 
l'environnement d'apprentissage peuvent en estomper les résultats, ou du moins rendre leur travail plus difficile. C'est du moins ce que semblent suggérer les études qui se sont penchées sur l'environnement d'apprentissage.

\section{Les variables en lien avec l'environnement d'apprentissage}

Les variables propres au contexte d'apprentissage constituent le dernier volet de ce tour d'horizon des facteurs relatifs à la participation verbale. Les recherches évoquent deux principales variables, soit la taille du groupe-classe ainsi que la disposition des bureaux et le positionnement spatial de l'étudiant.

5 Depuis l'arrivée massive d'étudiants vers l'enseignement universitaire, les enseignants sont confrontés à un nouveau problème : celui de la gestion de grands groupes. En effet, dans la plupart des pays, les ressources humaines octroyées à l'enseignement universitaire n'ont pas augmenté proportionnellement avec l'augmentation du nombre d'étudiants. Cela a eu un impact sur le ratio enseignant/étudiants qui a augmenté considérablement. Plusieurs enquêtes ont montré que l'augmentation de la taille des groupes d'étudiants est loin d'être anodine et que ses conséquences sont perceptibles tant chez les enseignants que chez les étudiants (Gibbs et Jenkins, 1992; Hargreaves, Galton et Pell, 1997; Swing et Peterson, 1981). Celles-ci dénoncent le caractère anonyme des classes, la difficulté à entrer en relation avec les enseignants et les autres étudiants, ainsi que la passivité que cela entraîne.

Crespo et Houle (1995) notent que dans une vaste salle de cours, les étudiants sont moins enclins à poser des questions et qu'ils restent souvent dans un rôle passif. Les enseignants soulignent, quant à eux, la difficulté à connaître les étudiants individuellement et à leur offrir une méthode d'enseignement appropriée. On a plutôt tendance à recourir à des méthodes traditionnelles (exposé magistral) pour faire face au nombre. De plus, la présence de grands groupes entraîne une raréfaction des rétroactions, une diminution des informations sur l'apprentissage et de l'aide personnalisée. La charge de travail de l'enseignant est nécessairement en lien avec le nombre d'étudiants inscrits au cours. Les conséquences d'une surcharge de travail peuvent se répercuter sur la quantité et la qualité des contacts avec les étudiants (PateBain, Achilles, Boyd-Zacharius et McKenna, 1992).

La taille du groupe-classe s'avère donc une variable importante de la participation verbale étudiante. Plusieurs recherches ont en effet confirmé le rôle essentiel joué par la quantité d'étudiants présents dans la classe (Auster et MacRone, 1994 ; Brady et Eisler, 1999 ; Canada et Pringle, 1995 ; Christensen, Curley, Marquez et Menzel, 1995 ; Crawford et MacLeod, 1990 ; Cornelius, Gray et Constantinople, 1990 ; Constantinople, Cornelius et Gray, 1988 ; Nunn, 1996). Ces auteurs observent que le nombre d'échanges verbaux entre étudiants et enseignant est inversement proportionnel à la taille du groupe-classe. Ainsi, plus le groupe est de petite taille, plus il est probable que le taux de participation verbale soit élevé. En d'autres mots, les petits groupes favorisent les interactions et les contacts enseignant-étudiants, en plus de rendre la gestion de classe plus facile (Slavin, 1989). Toutefois, les auteurs ne s'entendent pas sur les seuils pour discriminer les grands groupes des petits. Qui plus est, la plupart des études se sont penchées sur des groupes dont la taille varie entre 20 et 50 étudiants. Nous possédons 
donc très peu de données empiriques concernant les classes de plus de 50 étudiants ou celles de moins de 20 étudiants et de leur effet sur la participation.

Par ailleurs, la disposition du mobilier à l'intérieur de la classe semble avoir une influence sur la participation des étudiants. Les chercheurs Wong, Sommer et Cook (1992), après une étude qui s'est échelonnée sur 17 ans, concluent que les classes dont la disposition des tables de travail se présente en forme ovale, plutôt qu'en rangée, montrent des taux de participation verbale plus élevés. Ces auteurs révèlent aussi que dans les classes où les tables sont disposées en rangée, les étudiants qui s'assoient dans les premières rangées et au centre de la classe participent davantage que ceux assis à l'arrière ou vers les côtés. De plus, Rousvoal (2000) affirme que l'effet proxémique dû à la distance qui sépare l'enseignant des étudiants a un impact positif sur la réception des messages et les interactions verbales. Selon cet auteur, lorsque l'étudiant s'installe dans une salle de cours, l'endroit où il s'assoit est le reflet plus ou moins conscient de la relation communicationnelle qu'il entend avoir avec l'enseignant. De surcroît, il révèle que ce positionnement est relativement stable et que la majorité des étudiants (90,2\%) préfère s'asseoir approximativement au même endroit à chaque séance de cours. En effet, Rousvoal (2000) a constaté une régularité dans le positionnement spatial en salle et une très faible variation liée au type d'enseignement ou à l'enseignant. La plupart des étudiants sont capables de fournir une rationalisation à ce comportement. Par exemple, les étudiants qui choisissent de s'asseoir en avant se sentent plus concernés par le cours. Ils disent être mieux en mesure de voir et d'entendre ce que dit l'enseignant, cela leur procure un meilleur contact avec ce dernier. Ils peuvent plus facilement parler à l'enseignant et l'interrompre s'ils ont des questions. Ainsi, les étudiants du devant recherchent une meilleure qualité de réception du message pédagogique et manifestent la volonté d'interagir avec l'enseignant. Tandis que les étudiants qui choisissent les rangées du milieu recherchent plutôt une certaine neutralité. Ils disent s'y sentir plus à l'aise tout en se trouvant à une distance suffisante pour voir et comprendre l'enseignant sans être trop proche. En se positionnant au centre de la classe, ils ne visent qu'à être de simples récepteurs et peuvent facilement éviter de s'engager dans toute interaction avec l'enseignant puisqu'ils se fondent dans la masse. Enfin, les raisons évoquées par les étudiants qui s'assoient dans les rangées du fond de la classe sont moins nombreuses. Certains justifient leur positionnement par le fait qu'ils arrivent juste à l'heure et les rangées du devant sont toujours pleines. Pour d'autres, c'est une façon de limiter les interactions avec l'enseignant et d'être tranquille en cherchant à n'être que des observateurs de ce qui se passe dans la classe. De plus, la participation verbale en classe tend à fléchir du devant vers le fond de la classe (Rousvoal, 2000).

29 En résumé, les auteurs s'accordent pour dire que, généralement, les étudiants participent davantage à l'intérieur de groupes moins nombreux, alors que les groupes plus nombreux entravent la participation verbale. De plus, ils constatent que ce sont les étudiants qui s'assoient en avant de la classe qui participent le plus, mais que la disposition du mobilier peut s'avérer un élément qui joue sur la participation verbale en classe. Par ailleurs, on constate que l'environnement d'apprentissage a surtout été étudié en considérant des aspects physiques ou matériels de la salle de cours. À notre avis, il serait pertinent d'inclure des dimensions de nature plus affective, comme le climat de la classe, les règles tacites de communication ou les effets de la dynamique relationnelle. 


\section{Conclusion}

La présente revue de littérature s'est penchée sur les facteurs qui influencent la participation verbale en classe des étudiants en contexte universitaire. Les auteurs ont présenté les différents facteurs ayant fait l'objet de recherches antérieures, en les structurant à l'intérieur de trois catégories. La première catégorie réfère aux caractéristiques sociodémographiques et motivationnelles des étudiants, la seconde renvoie aux caractéristiques de l'enseignant et la troisième catégorie traite des caractéristiques de l'environnement d'apprentissage.

31 En dépit de certains résultats divergents, on constate que, dans l'ensemble, tous les facteurs étudiés peuvent avoir une influence sur la participation verbale. Toutefois, étant donné que la plupart des travaux ne s'attardent qu'à un ou deux facteurs simultanément, il est difficile de se prononcer sur leur importance relative et sur la nature des relations qui existent entre eux. À cet effet, il est nécessaire de poursuivre les études afin d'approfondir les connaissances liées aux facteurs en lien avec la participation verbale et pour acquérir des informations plus précises relativement aux liens entre les différents facteurs.

Ainsi, des études ultérieures devraient se pencher sur les effets principaux et combinés de ces facteurs. De plus, elles devraient introduire des variables qui n'ont pas fait l'objet d'études à ce jour, comme le climat de classe, les pratiques pédagogiques et évaluatives des enseignants, le nombre de sessions suivies et même le niveau d'anxiété des étudiants à parler en public. Ces dernières variables peuvent s'avérer importantes en termes d'effet qu'elles ont sur la perception des étudiants de leur environnement d'apprentissage et de leur comportement en classe.

33 Conséquemment, nous pourrions mieux connaître et comprendre les facteurs qui contribuent à la participation verbale et favoriser, de la sorte, le développement des compétences des étudiants. On peut aussi s'attendre à des retombées signifiantes pour le milieu universitaire, particulièrement en ce qui a trait aux pratiques pédagogiques et de l'organisation des environnements d'apprentissage à privilégier. De ce fait, un réaménagement des lieux d'enseignement et une affectation du nombre d'étudiants par classe qui tiennent compte des résultats de recherche pourraient sans doute favoriser la participation verbale des étudiants.

Cela dit, la participation verbale ne constitue pas une fin en soi. Néanmoins, comme le montrent les études citées dans ce texte, elle est souhaitable car elle peut mener à l'atteinte de plusieurs objectifs pédagogiques, dont l'apprentissage, la rétention et l'utilisation des connaissances. Elle peut également témoigner de l'engagement des étudiants dans leurs études et leur motivation à apprendre, qui constituent deux éléments importants de la réussite scolaire. 


\section{BIBLIOGRAPHIE}

Astin, A. (1984). Student involvement: A developmental theory for higher education. Journal of College Student Personnel, 25(4), 298-307.

Astin, A. (1993). What matters in college. San Francisco : Jossey-Bass.

Aitken, J. et Neer, M. (1992). The Relationship of Classroom Communication apprehension and Motivation to College Student Question-Asking. (service de reproduction de document ERIC No. ED347598).

Aitken, J. E. \& Neer, M. R. (1993). "College Student Question-Asking: The Relationship of Classroom Apprehension and Motivation." The Southern Communication Journal, 59,1, (pp. 74-81).

Auster, C.J. et MacRone, M. (1994). The classroom as a negotiated social setting: An empirical study of the effects of faculty members' behavior on students' participation. Teaching Sociology, 22(1), 289-300.

Bishop-Clark, C. et Lynch, J. (1992). The mixed age college classroom. CollegeTeaching, 40(3), 114-117.

Boersma, P., Gay, D., Jones, R., Morrison, L. et Remick, H. (1981). Sex differences in college student-faculty interactions: Fact or fantasy? Sex Roles, 8(1), 775-784.

Bradley, S. et Graham, S. (2000). The effect of educational ethos and campus involvement on selfreported college outcomes for traditional and nontraditional undergraduates. Journal of College Student Development, 41(5), 488-502.

Brady, K. et Eisler, R. (1999). Sex and gender in the college classroom : A quantitive analysis of faculty-student interactions and perceptions. Journal of Educational Psychology, 91(1), 127-145.

Bujold, N. et Saint-Pierre, H. (1996). Style d'intervention pédagogique, relations affectives enseignants-étudiants et engagement par rapport à la matière. La revue canadienne d'enseignement supérieur, 26(1), 75-107.

Canada, K.et Pringle, R. (1995). The role of gender in college classroom interactions: A social context approach. Sociology of Education, 68, 161-186.

Chan, B. et McCroskey, J. (1987). The WTC scale as a predictor of classroom participation. Communication Research Reports, 4(2), 47-50.

Chickering, A. et Gamson, Z. (1987). Seven principles for good practice in undergraduate education. AAHE Bulletin, 39(7), 3-7.

Chickering, A. et Ehrmann, S. ( 1996). Implementing the Seven Principles: Technology as Lever. AAHE Bulletin, 49(2), 3-6.

Chouinard, R. (2002). Différences d'attitudes et de comportement en classe selon l'appartenance sexuelle. In J. Fijalkow et T. Nault (dir.), La gestion de classe (pp.185-198). Bruxelles : De Boeck.

Chouinard, R. (1999). Enseignants débutants et pratiques de gestion de classe. Revue des sciences de l'éducation, 25(3), 497-514.

Christensen, L., Curley, K., Marquez, E. et Menzel, K. (1995). Classroom situations which lead to student participation. (service de reproduction de document ERIC No. ED391207). 
Conseil Supérieur de l'Éducation (1992). Les nouvelles populations étudiantes des collèges et des universités : des enseignements à tirer. Québec : Gouvernement du Québec.

Constantinople, A., Cornelius, R. et Gray, J. (1988). The chilly climate: Fact or artifact? Journal of Higher Education, 59, 527-550.

Cornelius, R., Gray, J. et Constantinople, A. (1990). Student-faculty interaction in the college classroom. Journal of Research and Development in Education, 23,189-197.

Crawford, M. et MacLeod, M. (1990). Gender in the college classroom: An assessment of the «chilly climate» for women. Sex Roles, 23(1), 101-122.

Crespo, M. et Houle, R. (1995). La persévérance aux études dans les programmes de premier cycle à l'Université de Montréal. Montréal, Québec : Université de Montréal.

Crombie, G., Pyke, S., Siverthorn, N., Jones, A. et Piccinin, S. (2003). Students' perceptions of their classroom participation and instructor as a function of gender and context. The Journal of Higher Education, 74(1), 51-76.

DeLucia, R. (1994). Perceptions of faculty-student relationships: A survey. NASPA Journal, 31(4), 271-279.

Fassinger, P. (1995a). Understanding classroom interaction. Students' and professors' contributions to students' silence. Journal of Higher Education, 66(1), 82-96.

Fassinger, P. (1995b). Professors' and students' perceptions of why students participate in class. Teaching Sociology, 24(1), 25-33.

Fritschner, L. (2000). Inside the undergraduate college classroom. Faculty and Student differ on the meaning of student participation. The Journal of Higher Education, 71(3), 342-362.

Gibbs, G. et Jenkins, A. (1992). Teaching large classes in higher education. London: Kogan Page.

Goldstein, G. et Benassi, V. (1997). Teacher self-disclosure and student classroom participation. Teaching of Psychology, 21(1), 212-217.

Hall, M. et Sandler, R. (1982). The classroom climate: A chilly one for women? Project on the status and education of women. Washington, D.C: Association of American Colleges.

Hargreaves, L., Galton, M. et Pell, A. (1997). The effects of major changes in class size on teacher-pupil interaction in elementary school classes in England: Does research merely confirm the obvious? Paper presented at the annual meeting of the american educational research association. Chicago, IL (service de reproduction de document ERIC No. ED409123).

House, D. (2000). The effect of student involvement on the development of academic self-concept. The Journal of Social Psychology, 140(2), 261-263.

Howard, J. et Henney, A. (1998). Student participation and instructor gender in the mixed-age college classroom. The Journal of Higher Education, 69(4), 384-405.

Howard, J., Short, B. et Clark, M. (1996). Students' participation in the mixed-age college Classroom. Teaching Sociology, 23, 8-24.

Karabenick, S. (2004). Perceived achievement goals structure and college student help seeking. Journal of Educational Psychology, 96(3), 569-581.

Karabenick, S. et Sharma, R. (1994). Perceived teacher support of student questioning in the college classroom: Its relation to student characteristics and role in the classroom questioning process. Journal of Educational Psychology, 86(1), 90-103. 
Karp, D. et Yoels, W. (1976). The college classroom: Some observations on the meaning of student participation. Sociology and Social Research, 60, 421-439.

Kozanitis, A. (1997). L'impact de la relation entre l'enseignant et l'étudiant sur la personne de l'enseignant et sur son enseignement. In Actes du $14{ }^{\text {ème }}$ congrès de l'Association internationale de pédagogie universitaire. Congrès tenu à Liège, Belgique.

Kozanitis, A. et Chouinard, R. (2007). Les effets directs des variables d'influence de la participation verbale en classe des étudiants universitaires. Revue canadienne de l'enseignement supérieur, 37(2), 1-28.

Kozanitis, A., Desbiens, J.-F. et Chouinard, R. (2007). Perception of teacher support and reaction towards questioning: Its relation to instrumental help-seeking and motivation to learn. International Journal of Teaching and Learning in Higher Education, 19(3), 238-250.

Kubanek, A.-M. et Waller, M. (1994). Poser des questions avec assurance. Pédagogie collégiale, 8(2), 13-17.

Kubanek, A.-M. et Waller, M. (1995). Une question de relation. Pédagogie collégiale, 8(4), 23-27.

Kuh, G. (2001). Assessing what really matters to student learning : Inside the national survey of student engagement. Change, 33(3), 10-17.

Kuh, G., Schuh, J. et Whitt, E. (1991). Involving colleges: Successful approaches to fostering student learning and personal development outside the classroom. San Francisco: Jossey-Bass.

Lamport, M. (1993). Student-faculty informal interactions and the effect on college student outcomes: A review of the literature. Adolescence, 28(112), 971-990.

Leet-Pellegrini, H. (1980). Conversational dominance as a function of gender and expertise. In H. Giles, P. Robinson et P. Smith (dir.), Language: Social Psychological Perspectives (pp. 97-104). Oxford: Pergamon.

Lynch, J. et Bishop-Clark, C. (1993). Traditional and nontraditional student attitudes toward the mixed age college classroom. Innovative Higher Education, 17, 109-121.

Menzel, K. et Carrell, L. (1999). The impact of gender and immediacy on willingness to talk and perceived learning. Communication Education, 48, 31-40.

Ministère de l'Éducation du Québec (1995). Indicateur de l'éducation. Édition 1995, Québec, Ministère de l'Éducation, Direction générale des ressources informationnelles.

Ministère de l'Éducation du Québec (2003). Statistiques de l'éducation. Enseignement primaire, secondaire, collégial et universitaire. Québec.

Nunn, C. (1996). Discussion in the college classroom. Journal of Higher Education, 67(3), 244-266.

Pace, C. (1995). From good practices to good products: relating good practices in undergraduate education to student achievement. Paper presented at the meeting of the Association for Institutional Research, Boston. (ERIC n ${ }^{\circ}$ ED 356 322).

Pajares, F. (1997). Current Directions in Self-efficacy Research. In M. Maehr \& P. Pintrich (Eds.). Advances in motivation and achievement. Volume 10, (p.1-49). Greenwich, CT: JAI Press.

Paoletti, G. et Rigutti, S. (2003). Question asking by writing anonymously in distance education. Communication présentée au dixième congrès de la European Association for Research on Learning and Instruction, Padoue, Italie.

Pascarella, E. et Terenzini, P. (1991). How college affects students. San Francisco : Jossey-Bass. 
Parmentier, P., Gathy, P., Paquet, F., De Ketele, J. et Denef, J. (1991). La gestion du temps comme facteur associé à la performance académique: présentation des résultats d'une enquête en première candidature de médecine à l'Université catholique de Louvain. Rapport de recherche, Université catholique de Louvain, Louvain-la-Neuve.

Pate-Bain, H., Achilles, C., Boyd-Zacharius, J. et McKenna, B. (1992). Class size makes a difference. Phi Delta Kappan, 74, 253-256.

Pearson, J. et West, R. (1991). An initial investigation of the effects of gender on student questions in the classroom : developing a descriptive base. Communication Education, 40(1), 22-30.

Pirot, L. et De Ketele, J.-M. (2000). L'engagement académique de l'étudiant comme facteur de réussite à l'université. Étude exploratoire menée dans deux facultés contrastées. Revue des sciences de l'éducation, 26(2), 367-394.

Plecha, M. (2002). The impact of motivation, student-peer, and student-faculty interaction on academic self-confidence (service de reproduction de document ERIC No. ED464149).

Ramsden, P. (2003). Learning to teach in higher education ( $2^{\text {nd }}$ ed.). London: Routlegde \& Falmer. Reynolds, K. et Nunn, C. (1997). Engaging classrooms: Student participation and the instructional factors that shape it (service de reproduction de document ERIC No. ED415815). Romainville, M. (2000). L'échec dans l'université de masse. Paris, L'Harmattan.

Rousvoal, J. (2000). Positionnement spatial de l'étudiant dans la salle d'enseignement et réussite en première année de D.E.U.G. L'orientation scolaire et professionnelle, 29(3), 519-545.

Sadker, M., Bauchner, J., Sadker, D. et Hergert, L. (1981). Observer's manual for INTERSECT : Interactions for sex equity in classroom teaching. Washington, DC: United States Department of Education.

Sadker, M. et Sadker, D. (1992). Ensuring equitable participation in college classes. New Directions for Teaching and Learning, 49, 49-56.

Sadker, M., et Sadker, D. (1986). Sexism in the classroom: From grade school to graduate school. Phi Delta Kappan, 67, 512-515.

Slavin, R. (1989). Class size and student achievement: Small effects of small classes.

Educational Psychologist, 24(1), 99-110.

Smart, J., Feldman, K. et Ethington, C. (2000). Academic disciplines: Holland's theory and the study of college students and faculty. Nashville, TN: Vanderbilt University Press.

Sternglanz, H. et Lyberger-Ficek, S. (1977). Sex differences in student-teacher interactions in the college classroom. Sex Roles, 3(4), 345-352.

Swing, S. et Peterson, P. (1981). The relationship of student ability and small-group interaction to student achievement. National institute of education, Washington, DC.

Tannen, D. (1995). The power of talk: who gets heard and why. Harvard Business Review, 73, 138-148.

Terenzini, P., Pascarella, E. et Blimling, G. (1996). Students' out-of-class experiences and their influence on learning and cognitive development: A literature review. Journal of College Student Development, 37(2), 149-162.

Terenzini, P., Pascarella, E. et Lorang, W. (1982). An assessment of the academic and social influences on freshman year educational outcomes. The Review of Higher Education, 5(2), 89-108. 
Thayer-Bacon, B. et Bacon, C. (1996). Caring professors: A model. The Journalof General Education, 45(4), 255-269.

Tinto, V. (1987). Leaving college: rethinking the causes and cures of student attrition. Chicago : University of Chicago Press.

Willis, D. (1993). Academic involement at university. Higher Education, 25(2), 133-150.

Wong, C., Sommer, R. et Cook, E. (1992). The soft classroom 17 years later. Journal of Environmental Psychology, 12(4), 337-343.

\section{NOTES}

1. Le terme enseignant est utilisé sans discernement quant au statut légal (professeur, chargé de cours, formateur, etc.) et réfère à toute personne qui offre une prestation d'enseignement auprès d'étudiants.

\section{RÉSUMÉS}

Les auteurs effectuent une revue de la littérature traitant des facteurs qui influencent la participation verbale en classe des étudiantes et des étudiants de premier cycle universitaire. Les divers facteurs ont été classés dans trois catégories et renvoient soit à des caractéristiques propres aux étudiants, à des caractéristiques relatives aux comportements des professeurs ou à des caractéristiques de l'environnement d'apprentissage. Cette catégorisation est une contribution originale des auteurs et a pour but d'offrir une structure cohérente de l'information.

The authors carried out a review of the literature on factors which influence undergraduates' in class verbal participation. Several factors were identified and classified in three categories: those focusing on student characteristics, those related to teacher's behavior, and those relevant to the learning environment. This categorization is an original contribution proposed by the authors in order to structure the body of knowledge.

\section{INDEX}

Mots-clés : caractéristiques étudiantes, comportements enseignants, contexte d'apprentissage, participation verbale, premier cycle universitaire

Keywords : learning context, students' characteristics, teacher behaviours, undergraduates, verbal participation 


\section{AUTEURS}

\section{ANASTASSIS KOZANITIS}

Conseiller pédagogique

Bureau d'appui pédagogique

École polytechnique de Montréal

C.P. 6079 , succ. Centre-ville

Montréal (Québec)

$\mathrm{H} 3 \mathrm{C} 1 \mathrm{~J} 4$

téléphone : (514) 340-4711 poste 3229

télécopie : (514) 340-3734

Courrier électronique : anastassis.kozanitis@polymtl.ca

\section{ROCH CHOUINARD}

Professeur titulaire

Département de psychopédagogie et d'andragogie

Université de Montréal

C.P. 6128 , succursale Centre-ville

Montréal (Québec)

H3C 3J7

Téléphone: :514) 343-6286

Télécopie : (514) 343-7660

Courrier électronique : roch.chouinard@umontreal.ca 